\title{
Effects of Electrodouble Layer (EDL) and Surface Roughness on Lubrication Theory
}

\author{
Wang-Long Li
}

Published online: 21 February 2008

(C) Springer Science+Business Media, LLC 2008

\section{Erratum to: Tribol Lett (2005) 20(1)}

\section{DOI 10.1007/s11249-005-7792-2}

Following are updates to Eq. 7c, Fig. 2b, and Figs. 3a and $b$, due to an error found in the derivation in the original article:

The correct coefficient in Eq. (7c) is $A_{3}^{\prime}=6+$ $\frac{2 \eta_{e}}{\eta} \frac{\kappa h(\cosh \kappa h-1)^{2}}{\sinh ^{3} \kappa h}$. The expressions of coefficients $g_{x}$ (Eq. 6b), pressure flow factors $\phi_{x x}^{p}$ (Eq. 4a), and $\phi_{y y}^{p}$ (Eq. 4b), and shear flow factors $\phi_{x x}^{p}$ (Eq. 4e) and $\phi_{y y}^{p}$ (Eq. 4f) are correct in the original article. However, they all are related to $A_{3}^{\prime}$; therefore, the calculated value should be corrected along with the correction of $A_{3}^{\prime}$.

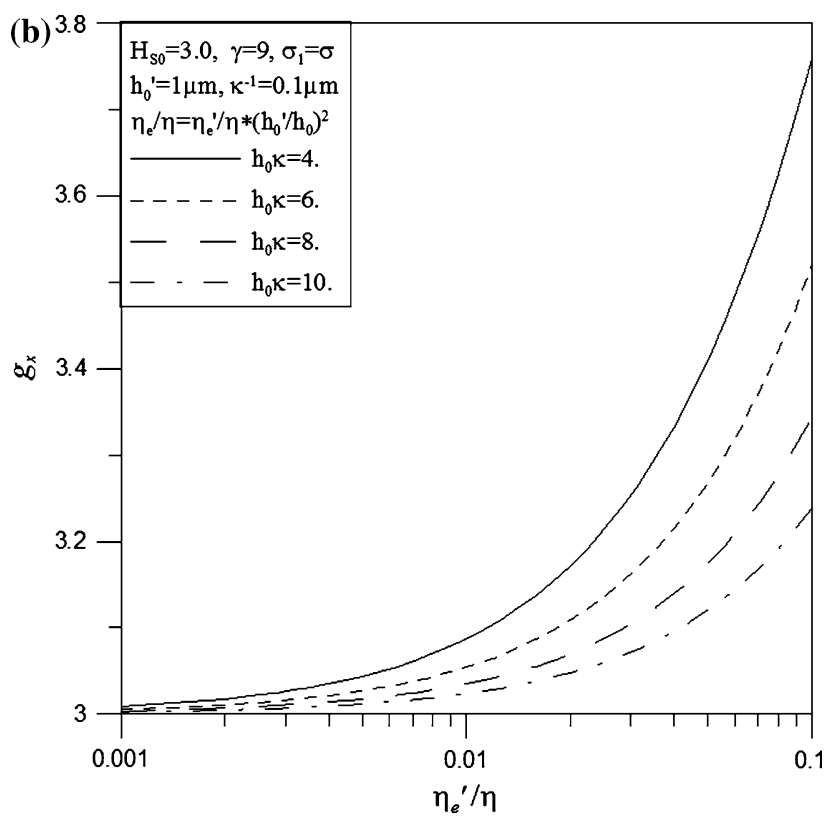

Fig. 2b
The online version of the original article can be found under doi: 10.1007/s11249-005-7792-2.

\section{W.-L. Li ( $\square)$}

Institute of Nanotechnology and Microsystems Engineering, Center for Micro/Nano Research Center, National Cheng Kung University, No.1 University Road, Tainan 701, Taiwan

e-mail: wlli@mail.ncku.edu.tw 

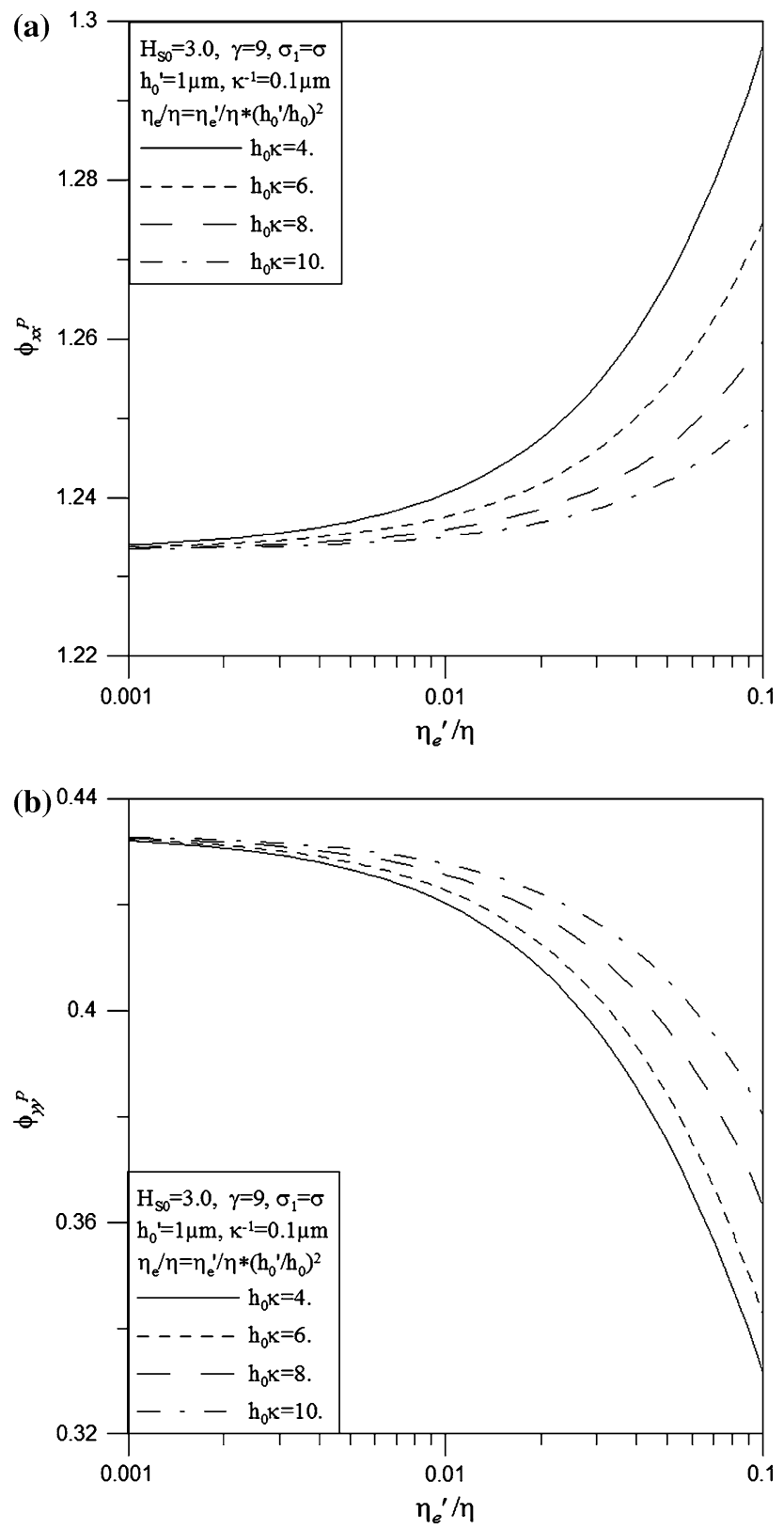

Fig. 3a, b 